\title{
Mujer y trabajo: transformaciones familiares tras su incorporación al escenario laboral*
}

Cómo citar este artículo:
Cortés, L., Ruiz, M. J. y Flórez, G. (2021).
Mujer y trabajo: transformaciones familiares
tras su incorporación al escenario laboral.
Revista Latinoamericana de Estudios de
Familia, 13(2), 78-100.
https://doi.org/10.17151/rlef.2021.13.2.5

\author{
Liliana Cortés-Rodas** \\ María José Ruiz-Turizo*** \\ Giovanny Flórez-Marín****
}

Recibido: 5 de enero de 2020

Aprobado: 4 de mayo de 2020

\begin{abstract}
Resumen: Este artículo de revisión tuvo como objetivo identificar las transformaciones en las familias con el ingreso de la mujer al escenario laboral. Su metodología partió de la revisión documental sistemática de estudios realizados entre 1980 y 2018. Como resultado se ubicaron cuatro categorías: 1 ) Autonomía de la mujer y nacimiento de la doble jornada, 2) Te colaboro, pero no me toca: hombres por obligación y mujeres en la tradición, 3) Cambios tras las resistencias y 4) Surgimiento de nuevas paternidades en el hogar. Finalmente, se concluye que las transformaciones familiares con la incorporación de las mujeres al escenario laboral están relacionadas con nuevas configuraciones familiares democráticas e igualitarias, el liderazgo y autoridad compartida, donde se generan procesos de negociación más flexibles. Además, se hallaron cambios en el cuidado, evidenciando disconformidad masculina, pero donde los hombres empiezan a participar de las tareas del cuidado, adoptando la construcción de una paternidad afectiva.
\end{abstract}

Palabras clave: mujer, trabajo, familia, transformación, paternidad.

\footnotetext{
* El presente artículo de investigación es el producto de opción de grado II para optar al título de Trabajadoras Sociales de la Corporación Universitaria Minuto de Dios, Antioquia (Colombia).

** Corporación Universitaria Minuto de Dios. Bello, Colombia. E-mail: Icortesroda@uniminuto.edu.co.

(iD orcid.org/0000-0002-7523-5790. Google Scholar

*** Corporación Universitaria Minuto de Dios. Bello, Colombia. E-mail: mruizturizo@uniminuto.edu.co.

(D) orcid.org/0000-0002-1347-8885. Google Scholar

Corporación Universitaria Minuto de Dios. Bello, Colombia. E-mail: giovanny.florez.m@uniminuto.edu.

(iD) orcid.org/0000-0002-6878-8207. Google Scholar
} 


\title{
Woman and work: family transformations after entering the work scene
}

\begin{abstract}
The objective of this review article is to identify the transformations in families with the entry of women into the work scenario. Its methodology was based on the systematic documentary review of studies carried out between 1980 and 2020. As a result, four categories were found: 1 ) Women's autonomy and the birth of the double shift; 2) "I will help you, but it is not my turn": men by obligation and women in tradition; 3 ) changes after resistance; and 4) emergence of new paternities in the home. Finally, it is concluded that family transformations with the incorporation of women into the work scene are related to new democratic and egalitarian family configurations, leadership and shared authority, where more flexible negotiation processes are generated. In addition, changes were found in caregiving, evidencing male disagreement, but where men begin to participate in caregiving tasks, adopting the construction of an affective fatherhood.
\end{abstract}

Key words: woman, work, family, transformation, parenthood.

\section{Introducción}

Un punto de partida para abordar las transformaciones familiares con la incorporación de la mujer al ámbito laboral es la perspectiva de Morgan (citado en Engels, 1891), quien subrayó la influencia de la sociedad sobre la forma y la estructura de la familia, señalando que esta "es el elemento activo; nunca permanece estacionada, sino que pasa de una forma inferior a una forma superior a medida que la sociedad evoluciona de un grado más bajo a otro más alto" (p.2), considerando que la familia no es una unidad estática, sino que está en un cambio continuo igual que los contextos sociales, reflejado en su cultura e influida por cambios socioeconómicos y patrones idiosincráticos de cada región (González, 2008).

Teniendo presente este movimiento y evolución de la familia, durante las últimas décadas la sociedad ha evidenciado cómo estas se han transformado siendo el resultado de los procesos de industrialización, modernización y desarrollo económico. Dichos avances de la modernidad han traído, en el caso específico de la mujer, sustanciales cambios en los niveles de escolaridad, acceso a los servicios de planificación 
familiar y cambios culturales sobre los roles femeninos que han incrementado su participación en el mercado de trabajo y las funciones que desempeñaba en el ámbito familiar (García y De Oliveira, 2001; Cásares, 2008; Veneros y Ortega, 2011; Urrutia y Figueroa, 2015; Duarte y García-Horta, 2016).

Frente a los cambios de la mujer en la modernidad, Santelices et al. (2015) afirman que dichas transformaciones económicas, sociales y políticas han llevado a las mujeres a cambiar sus roles tradicionales, ya sea por elección o por necesidad, generando que las mujeres cambien actividades del hogar por el ámbito laboral, aun cuando estas han estado históricamente vinculadas a las prácticas maternales (cuidado, alimentación, limpieza, procesos de socialización y aprendizaje, entre otras) y funciones que han sido determinadas y segregadas según su género y condición reproductiva (Tobio, 1998; Sabater, 2014; Urrutia y Figueroa, 2015; Romero, 2018).

En el marco del contexto histórico, es útil recordar que desde tiempos vetustos se identifica a la mujer vinculada a las actividades relacionadas con la preparación del alimento, raspar la piel de animales, elaborar prendas de vestir, el cuidado del fuego, entre otras actividades del escenario doméstico (Duarte y García-Horta, 2016; Romero, 2018). En la Edad Media la mujer quedaba relegada al centro del hogar, del que era dueña y señora, atendiendo tareas como la crianza de los hijos, el aspecto educación, la organización del servicio y para algunas la economía del hogar en ausencia del marido. La mujer campesina de aquella época era la encargada de las tareas domésticas, la educación de los hijos y actividades como el cuidado de las huertas, animales para la producción, elaboración de conservas y comercialización de los productos (Labarge, 2003).

Para la época del Renacimiento, la maternidad era su profesión y su identidad, era un ciclo continuo de embarazo y crianza. Las mujeres estaban forzadas a ser fértiles y a dedicarse a la procreación y crianza de los hijos como cualidad principal de la mujer, además como sinónimo de prosperidad familiar (Duarte y García-Horta, 2016).

Valdivia (2008) destaca que la era moderna trae consigo la formación de familias tradicionales, donde se pretendía establecer que la mujer desempeñara acciones diferenciadas del hombre, ubicándola nuevamente en el ámbito de lo privado y desempeñando tareas en la atención y el cuidado de las personas, la cocina, la costura y la compra de ropa, la educación religiosa y transmisión de creencias. En pocas palabras, todas las funciones asociadas a ser madre y esposa reproduciendo la visión tradicional.

Dejando de lado esta visión tradicional de la maternidad y de la mujer, justamente en el tránsito de lo moderno a lo contemporáneo, nos encontramos con mujeres que participan activamente dentro de las actividades económicas, científicas y culturales, espacios que antes solo estaban abiertos para los hombres (Mato, 1999). 
Actualmente, las mujeres desempeñan multiplicidad de roles de gran exigencia que van desde las labores domésticas, contención emocional de sus familias y carga laboral y económica que le significa el trabajo extradoméstico, construyendo modelos diferentes de "ser mujer". Estas nuevas dinámicas sociales han llevado cambios en las esferas más significativas de la vida de estas mujeres, dado que ahora ejercen una participación social diferente siendo madres y esposas de tiempo completo, sumado a ocupar un lugar en el ámbito público (Rincón, 1995; Caballero, 2017).

Es así, con el trascurrir del tiempo, como la incorporación de la mujer del ámbito doméstico pasa a estar en el ámbito público, sumándose al mercado laboral y, aunque ha sido un proceso lento, lo cierto es que cada vez aumentan sus posibilidades de acceder a determinados sectores que antes estaban "prohibidos" (Vega, 1994; Pérez-Baleón, 2012). Esta inclusión ha generado modificaciones importantes en la propia realidad de ellas y la de sus familias, suponiendo retos para las mujeres que ahora dirigen hogares, son responsables de la producción, reproducción, cuidado y crianza de hijos, en tanto los hombres han comenzado también su tránsito.

\section{Metodología}

El presente artículo es producto de la revisión documental que sintetiza los resultados de múltiples investigaciones y artículos científicos, donde se seleccionaron algunos estudios relevantes que den respuesta a la pregunta de investigación y centre su interés en exponer el fenómeno social de la incorporación de la mujer al ámbito laboral y su relación con las transformaciones que se han experimentado en el sistema familiar en las últimas décadas.

\section{Método}

Este estudio pretende aproximarse a una de las realidades más relevantes que presenta la sociedad actual. El estudio se desarrolló bajo un enfoque cualitativo, el cual permite interpretar y reflexionar sobre las realidades sociales y acercarse a ellas, con el fin de ampliar las visiones, darles sentido a dichas realidades y construir conocimiento. Según Vargas (2011): "La metodología cualitativa es aquella cuyos métodos, técnicas, estrategias e instrumentos concretos se encuentran en lógica de observar necesariamente de manera subjetiva algún aspecto de la realidad" (p. 21), interpretando todos estos sucesos inmediatos a la luz de experiencias anteriores y de cualquier elemento que pueda ayudar a entender mejor la situación estudiada. 


\section{Muestra}

Para el desarrollo del artículo se rastrearon en total 60 documentos científicos, verificando su pertinencia para este estudio, sin embargo, se seleccionaron 55 de ellos que aportaron información relevante para la construcción de este artículo. Los artículos fueron buscados en las bases de datos: Google Académico, Repositorio biblioteca virtual UNIMINUTO, Scielo, Dialnet, JSTOR, ResearchGate, Redalyc, EBSCO, STOR, Journals, Proquest y Oxford Academic. Es significativo plantearse que la mayor información encontrada en relación con la temática fueron investigaciones de países como Argentina, Brasil, Chile, Colombia, Costa Rica, México y USA.

\section{Estrategia/Técnica}

La estrategia elegida para el desarrollo del artículo, como ya se mencionó, fue la investigación documental, la cual se basa en la pesquisa de documentos digitales entre investigaciones y artículos científicos realizados entre los años 1980 y 2018, entendiendo que después de los años 70 se gestó la segunda ola feminista o feminismo de la liberación que avecinaba cambios en la relación a la mujer frente a los escenarios laboral, familiar y las concepciones tradicionales (Lamus, 2010). Es desde este lugar que surgen las temáticas de mujer, trabajo, familia y sus transformaciones; estas palabras clave permitieron delimitar la búsqueda de información. Ampliando el significado de esta modalidad de investigación, es pertinente plantear que la investigación documental tiene un carácter particular donde se presenta como cualidad el aspecto interpretativo, sumado a leer y otorgar sentido a los textos que fueron escritos con una intención distinta pero que al final se le dan atributos comprensivos (Vargas, 2011).

\section{Instrumentos}

Para el registro, el análisis y la interpretación de la información encontrada se utilizaron fichas bibliográficas, matrices de análisis y matrices de interpretación, las cuales permitieron la extracción de apartados de las investigaciones que se consideraron importantes para el momento del análisis y el desarrollo de las categorías principales y categorías emergentes, donde posteriormente se analizan los resultados para generar la discusión y las conclusiones.

\section{Resultados}

Los resultados que se presentan a continuación son el resultado del análisis de los documentos revisados e interpretados. Este proceso arrojó las siguientes categorías: 
1. Autonomía de la mujer y nacimiento de la doble jornada.

2. Te colaboro, pero no me toca: hombres por obligación y mujeres en la tradición.

3. Cambios tras resistencias.

4. Surgimiento de nuevas paternidades en el hogar.

\section{Autonomía de la mujer y nacimiento de la doble jornada}

Esta categoría hace referencia al proceso de autonomía e independencia económica logrado por la mujer a través de la lucha femenina por el reconocimiento de sus derechos, la búsqueda de igualdad, el acceso a la educación y el logro por obtener un trabajo remunerado. Asimismo, esta categoría refleja los cambios a nivel individual y las transformaciones familiares en las relaciones, la reorganización de las dinámicas de género, los roles y el surgimiento de la doble jornada femenina.

Autores como Covarrubias (2012) expresan que las mujeres que deciden trabajar logran independencia económica, puesto que son productivas laboralmente y esto les genera una autonomía necesaria para modificar algunas normas y prácticas familiares. En la misma línea, Guillén (2007) y De Oliveira y Ariza (1999) sostienen que hay una relación positiva entre la actividad extradoméstica femenina y la toma de decisiones, en la cual se han empezado a construir espacios de acción y libertad donde a las mujeres, al tener acceso a una serie de recursos producto de su actividad laboral, se les ha permitido cuestionar patrones de autoridad y modificar su condición de subordinación, empezando a crear identidades de género más autónomas y relaciones más simétricas dentro del hogar.

Sin embargo, Gómez y Jiménez (2015) manifiestan que dicha actividad extralaboral lejos de distanciarla de su rol tradicional reproductor, se ha convertido en una doble carga para ella y aunque se reconocen como mujeres-madres-esposas y trabajadoras a la vez, su rol familiar y de cuidado de los hijos no cambia porque continúan con toda la responsabilidad. Esto hace visible la sobrecarga de obligaciones cotidianas de las mujeres, quienes ahora no solo desempeñan una actividad extradoméstica, sino que también la combinan con tareas representativas de su género, que no son compartidas con la figura masculina (De Oliveira y Ariza, 1999).

Lo expresado anteriormente sugiere que, aun cuando las mujeres sienten un alto nivel de autorrealización con su trabajo productivo, se enfrentan a dificultades en el ámbito familiar, ya que interactúan de forma paralela entre las actividades laborales y las actividades domésticas, lo que puede llevar a conflictos relacionados con la conciliación trabajo-familia y la sobrecarga del rol (Rodríguez y Fernández, 2010). En esa misma línea, Pleck (2014) y Sabater (2014) narran que las mujeres han aumentado su participación laboral como proveedoras económicas, a la vez que han 
disminuido su capacidad de cuidado en el espacio doméstico, pero hasta ahora no se identifica un aumento en el papel familiar de los esposos.

En lo que respecta a la decisión de trabajar para el caso de algunas mujeres, Rodríguez y Fernández (2010) señalaron que no es la necesidad o la condición socioeconómica el factor motivador que las lleva a ingresar al trabajo productivo, sino sus propias convicciones y el significado que le dan al trabajo en sí mismo, el cual es una forma más de realización personal sumado a la manera de suscitar cambios sobre las percepciones de sus hijos en relación a la mujer. Por el contrario, autores como García et al. (1982), Haller y Hoellinger (1994) y Abramo (2004) coinciden que cuando se da la inserción laboral de la mujer su proyecto de vida es secundario a crisis económicas, desempleo, enfermedad, edad avanzada del jefe de la familia o ausencia de la figura masculina resultado de viudez o separación por lo que la mujer debe asumir el rol económico del hogar casi que por obligación.

Wollstonecraft y Friedan (citados en Duarte y García-Horta, 2016) indican que la lucha de las mujeres por la igualdad no solo se define por su participación activa e igualitaria en procesos políticos, sociales y económicos, de los que si bien es cierto han surgido diversos avances en el reconocimiento de sus derechos, estos aún no han sido aplicados de manera general en otros ámbitos. Además de estos aspectos, se encuentra la reorganización y división equitativa de las responsabilidades dentro del hogar en lo que respecta a los hijos y a las diferentes tareas domésticas, que permitan un equilibrio entre la actividad laboral remunerada y el hogar (Medina et al., 2005).

En concordancia con la reorganización y división equitativa de las responsabilidades del hogar, Spitze (1988) y García y De Oliveira (1994) expresan que los hombres, en general, dedican menos tiempo a las tareas domésticas que las mujeres, así estas estén o no empleadas extralaboralmente, siendo uno de los aspectos que ha experimentado menos cambios dentro del hogar, puesto que la responsabilidad del trabajo doméstico sigue siendo de la mujer, demostrando que aún con su ingreso al mercado laboral las mujeres mantienen su rol tradicional, sumado a generar aportes económicos para la misma. En este contexto se reconoce que, si bien la participación económica de la mujer no es una condición suficiente para el logro de una división equitativa de responsabilidades en el hogar, se ha avanzado en la definición de algunas posibles transformaciones en las relaciones familiares. Autores como Bonaccorsi (1999) y Ayala et al. (2011) refieren que la sociedad no considera a la mujer trabajadora en su doble papel, dado que se ignora el trabajo doméstico de esta, y solo se estiman las labores en términos de producción y rentabilidad. Este relacionamiento de la doble jornada se puede generar porque las mujeres han naturalizado el cuidado como un asunto netamente femenino y son ellas mismas las que no incorporan al género masculino en dicho proceso (Tirado et al., 2016). 
García y De Oliveira (1994) consideran que la contribución femenina al presupuesto familiar incide en su participación en la toma de decisiones, el control de su reproducción y los derechos que esta considera relevantes en relación con su vida. Asimismo, otros autores indican que el trabajo extradoméstico puede impulsar a las mujeres a elevar su autoestima y a lograr mayor respeto y espacios mínimos de control en el interior de sus familias, sosteniendo que las mujeres que se incorporan al mercado de trabajo consiguen mayor valoración de sus familias, autorrealización e independencia; a su vez, resaltan el progresivo cambio a un tipo de liderazgo participativo avanzado hacia escenarios más igualitarios donde la mujer tiene voz y participa activamente en la toma de decisiones (Philip, 2002; García y De Oliveira, 2004; Navarro, 2010).

Por otra parte, queda en evidencia cómo el ingreso de las mujeres al ámbito laboral conlleva a una transformación de las relaciones familiares tradicionales, creando resistencias por parte de las familias, sobre todo en las relaciones de pareja en lo que tiene que ver con la autonomía e independencia de las mujeres (Fawaz y Soto, 2012). Asimismo, se sugiere una visión en la cual la incorporación de la mujer al ámbito laboral constituye una condición necesaria para la equidad, es decir que, si la mujer tiene acceso a la economía, su posición de poder y participación dentro de la familia aumentará en la misma medida (Vega, 1994). Bajo este mismo esquema, Arriagada (2004) plantea la autonomía femenina como un elemento clave para un cambio en las relaciones internas de las familias, sobre todo en los procesos de reparto de las tareas domésticas y eficacia de los padres ante las necesidades de los hijos, permitiendo que hombres y mujeres adopten nuevas formas de organizar sus responsabilidades familiares y laborales.

Finalmente,Balbo (citado en Bonaccorsi,1999) sostiene que obtener autonomía no implica que la mujer renuncie a sus responsabilidades reproductivas y familiares. La autonomía hace parte de la capacidad de conciliar estos dos ámbitos, avanzando en su participación en el ámbito público y resignificando el ámbito doméstico. Siguiendo con esta misma idea, Urrutia et al. (2017) manifiestan que cuando la mujer incursiona en el mercado laboral se da la construcción de configuraciones familiares más democráticas, nuevos procesos de negociación frente a las tareas del hogar y las responsabilidades laborales al igual que cambios en la organización del cuidado. Además, se destacan las transformaciones en la toma de decisiones, donde ambos cónyuges deciden o no sobre el número de hijos que desean y su crianza, así como cambios acerca de quién asume la máxima autoridad en cuestiones importantes (García y De Oliveira, 1994). Sin embargo, también indican que las transformaciones en la vida familiar aún no han alcanzado un nivel de equidad y, si bien hay un avance en el curso de vida, la transición es muy lenta y aún predominan condiciones e imaginarios que hacen a las mujeres únicas responsables de las tareas tradicionales familiares. Es por esta razón que el papel integral de las mujeres modernas es complejo 
y trae consigo dificultades y tensiones familiares para lograr el equilibrio entre ambas esferas, dejando ver conflictos sobre todo en lo que tiene que ver con la gestión del tiempo. Hoy las mujeres se han convertido en socias del hogar, deben ganarse la vida y a su vez trabajan para cumplir con las responsabilidades como esposa, madre y ama de casa y aun así han encontrado formas para compensar su ausencia en el hogar, los hijos se sienten orgullosos de ellas y se identifican efectos beneficiosos en sus relaciones conyugales (White, 1999; Gómez y Marti, 2004).

\section{Te colaboro, pero no me toca: hombres por obligación y mujeres en la tradición}

El concepto te colaboro, pero no me toca: hombres por obligación y mujeres en la tradición, explora la relación entre la incorporación de las mujeres al mercado extradoméstico y las transformaciones en la división del trabajo. A su vez, aborda las diferencias existentes en la participación de hombres y mujeres en el entorno doméstico, situación que conlleva a conflictos familiares por el desempeño de los roles.

Históricamente, el reparto de tareas familiares (reproductivas-productivas) entre hombres y mujeres ha estado condicionado según el sexo biológico. Sin embargo, circunstancias sociales y económicas, tales como la entrada masiva de las mujeres al mercado de trabajo, han implicado unos cambios en la relación entre familia y trabajo. Dichos cambios sugieren que la mujer no puede seguir manteniendo las mismas funciones que venía realizando, además de trabajar fuera del hogar, sin que los hombres deban cambiar también, haciéndose necesario un nuevo reparto de las funciones y responsabilidades familiares entre los cónyuges (Gómez y Marti, 2004; Gómez y Jiménez, 2015).

Al respecto, Ribeiro (2004) expresa que el rol tradicional de la mujer parece aún no transformarse con su participación en el ámbito laboral, dado que los esposos y compañeros insisten en considerar que las tareas del hogar son responsabilidad de las mujeres y, cuando un hombre hace alguna de ellas, está ayudando a su pareja como un acto de favor y no de responsabilidad familiar. Esto indica que, si bien existe una masiva incorporación de la mujer en el mercado laboral compartida con los hombres, el cambio del modelo tradicional de único proveedor masculino ha dado paso a otras formas de ordenamiento familiar, donde aún persisten diferencias significativas según el género, en el cual las mujeres continúan asumiendo mayoritariamente el trabajo reproductivo (White, 1999; Ribeiro, 2004).

Frente a estos movimientos de género, que se pueden visibilizar en los roles que hombres y mujeres desempeñan, es significativo reconocer cómo el rol productor de la mujer ha propiciado transformaciones en las formas como se organizan las familias, lo que ha implicado simultáneamente una mayor participación de los hombres en oficios que tradicionalmente no realizaban, y una reestructuración en el reparto de 
las tareas domésticas y responsabilidades en el hogar. Sin embargo, se reconoce que las conductas de estos hombres no son las esperadas y dicha participación masculina en los trabajos domésticos se ha venido dando con un carácter de "ayuda", y son las mujeres quienes siguen teniendo una mayor carga de trabajo (Macías et al., 2000; Tirado et al., 2016).

Googins y Burden (1987) deducen que la disposición de cambio y de adaptación aún está permeada por creencias culturales que refuerzan estereotipos y roles de género tradicionales y que disminuyen la apertura de actitudes masculinas más participativas frente a las nuevas demandas familiares. Además, se evidencia que aún coexiste una ideología de desigualdad que permea las prácticas cotidianas de trabajo doméstico, dificultando la negociación y conllevando a un ejercicio efectivo de corresponsabilidad. Los hombres todavía ayudan, colaboran o escogen aquellas tareas que les resultan más llevaderas contribuyendo a la persistencia de prácticas asimétricas (Urrutia et al., 2017).

Por otro lado, se hace necesario resaltar que los hombres, sobre todo los más jóvenes, empiezan a apoyar el cambio hacia la igualdad, aunque esto no se traduzca significativamente en un compromiso constante por hacerse cargo de la mitad del trabajo doméstico y familiar (Vega, 1994). Igualmente se considera que, pese a estas posiciones menos tradicionalistas en términos de género, la flexibilización de los roles y la apertura de negociaciones para distribuir las tareas domésticas no son tan objetivas, puesto que la actuación del hombre dentro del hogar es vista como una colaboración de apoyo a la mujer, y no como tareas propias de su rol (Gómez y Jiménez, 2015; Urrutia et al., 2017). En efecto, en este contexto de falta de armonización, entre los cambios producidos en el espacio productivo y reproductivo con el acceso de la mujer al ámbito extradoméstico, se puntualiza en que son las mujeres a quienes les cuesta delegar funciones y se empeñan en asumir directamente todo el trabajo, además son muy exigentes en cuanto al nivel de desempeño en lo doméstico, obstaculizando la transformación del rol masculino.

Sin embargo, es significativo evidenciar cómo los hombres están cambiando dado qué no tienen otra salida, al incrementarse la presencia de las mujeres en el mercado de trabajo; es decir que el hombre se ha visto presionado a participar de los roles tradicionales de la mujer ante su participación activa en la esfera pública, dejando ver que el grado de participación masculina en la resolución de tareas típicamente femeninas no depende solo de la implicación de la mujer en el mercado de trabajo, sino también de la remuneración que a través de su trabajo recibe, ya sea en términos de status o monetarios. Cuanto mayor es la remuneración que la mujer obtiene de su implicación en el mercado de trabajo, mayor es la participación masculina (Landwerlin, 1997; García y De Oliveira, 2001; Amarís, 2011).

En otro frente se ha planteado que, si bien el empleo femenino extradoméstico tiende a generar cambios relevantes a nivel funcional, también ha contribuido 
significativamente a la desestabilización del sistema familiar (Philip, 2002). Esta idea también es sostenida por Spitze (1988) y Gómez y Marti (2004), quienes afirman que la participación de la mujer en trabajos fuera del hogar ha afectado su función principal como responsable del hogar, cambios que trascienden a conflictos para mantener el equilibrio entre la dedicación a la familia y al trabajo. Estos resultados sugieren que las mujeres que deciden ingresar a la esfera productiva no abandonan la esfera reproductiva, por tanto, la suma de responsabilidades familiares-laborales comienzan a tornarse en un obstáculo, ya que les resta tiempo de dedicación a la familia. Sin embargo, estos mismos autores señalan que esta situación puede generar tensiones y conflictos familiares y de pareja si no hay una conciliación familiar que genere un equilibrio de roles; la poca implicación del hombre en el trabajo familiar puede significar una ruptura conyugal o la interrupción del trabajo remunerado femenino (Philip, 2002; Gómez y Jiménez, 2015).

\section{Cambios tras resistencias}

Esta categoría hace referencia a las resistencias que presentan algunos hombres en relación con el cumplimiento de las funciones dentro de la familia, evidenciando además la génesis de los conflictos familiares, dado que no en todas las familias se logra una conciliación en los roles de familia. De forma breve, lo que se visibiliza en este apartado es la necesidad de una reacomodación familiar, donde los hombres no solo hagan parte de esa esfera pública, sino que además se involucren en la esfera privada contribuyendo de distintas formas en el hogar.

Vega (1994) manifiesta que la decisión de participación laboral para la mujer es de carácter opcional o coyuntural y, aunque se ha logrado cierto grado de aprobación y flexibilización de los condicionantes familiares, esta sigue considerándose la encargada principalmente de atender las necesidades de los hijos, sobre todo en las primeras etapas de vida. Esto evidencia cómo los hombres siguen estando relegados en la modernidad del ámbito doméstico.

De otro lado, De Oliveira y Ariza (1999) manifiestan que las transformaciones ligadas a la conformación familiar han sido factores determinantes en el aumento del número de mujeres que ingresan al mundo laboral, sumado a la manera en que las familias se adaptan para permitir su ingreso. Es en este punto donde la clara participación de las mujeres en el trabajo remunerado, específicamente las casadas, ha contribuido a provocar cambios en los papeles conyugales, y ha llevado a la familia a replantearse las ideas que tienen sobre el modelo tradicional y las nuevas formas de vinculación y orden familiar (Ribeiro, 2004). En esa misma línea, De Oliveira y Ariza (1999) señalan que las mujeres solteras tienen más facilidades a la hora de encontrar un trabajo que aquellas que están casadas o tienen hijos pequeños, dado que pueden acceder a diferentes formas de contratación puesto que no tienen 
limitaciones relacionadas con la conciliación de vida familiar/laboral, sin embargo, si tuvieran familia o pareja esto representaría una dificultad a conciliar a diferencia de los hombres en este mismo rol.

Frente al campo de las transformaciones familiares, Sabater (2014) señala que uno de los cambios que más influye en la familia es la mutación de los roles entre hombres y mujeres, evidenciando cómo el aumento del empleo femenino obliga a las familias a buscar nuevas alternativas de organización, que solo pueden ser alcanzadas con procesos de negociación, aunque encontrar el equilibrio puede tardar varios años. Además, se deja en claro que cuando la mujer no estudia y no participa en el mercado de trabajo es tenida en cuenta solo para estar presente en el trabajo doméstico y todo lo que este requiere, no visibilizándola para desempeñar otras labores (García et al.,1982).

Asimismo, Sabater (2014) expresa que la disminución en las tasas de natalidad puede deberse a un intento de conciliación de la vida laboral y familiar por las mujeres, retrasando o incluso abandonando la formación de un hogar y la maternidad, por ser vista como la principal barrera para alcanzar una vida profesional y laboral plena. Desde una visión tradicional, muchas mujeres no han buscado crecer a nivel individual por dedicarse a lo doméstico y/o familiar, y al llegar oportunidades laborales, si no ha existido una repartición de responsabilidades con el cónyuge, esto traerá cambios significativos dentro del núcleo familiar que necesitará una reacomodación de funciones y responsabilidades (Bonaccorsi, 1999). Asimismo, White (1999) plantea que las mujeres deben constituirse y replantearse dentro de un marco de relaciones igualitarias, donde sus actitudes y expectativas favorezcan su relación trabajo-familia, con el fin de no continuar generándose sentimientos de culpa por no responder a las expectativas tradicionales.

Complementando lo anterior, Fawaz y Soto (2012) manifiestan que los cambios en el hogar con la progresiva participación de la mujer en el escenario laboral no se han dado de la misma manera que los hombres en el aspecto laboral, dado que la división de roles ha requerido de una reestructuración general familiar que permita la conciliación de estos dos ámbitos, y en el caso de los hombres este proceso se ha dado más sencillo.

De otro lado, se ha generado un movimiento significativo a nivel laboral para las mujeres, donde se les reconocen espacios como lo maternidad, lo cual visibiliza flexibilidad y lograr el equilibrio frente a las dinámicas femeninas y masculinas. Sin embargo, dicha flexibilización laboral también exige flexibilización doméstica, pues se ha evidenciado que no todos los hombres han entendido sus responsabilidades en el ámbito doméstico e inclusive mujeres en situaciones delicadas de salud posparto han asumido responsabilidad en el hogar que requieren mayor compromiso de sus parejas (Landwerlin, 1997). 
En el aspecto de flexibilización, es trascendental visibilizar cómo se han logrado avances en el reparto de funciones en el hogar aunque aún hay resistencias con la incursión de la mujer en el ámbito laboral, pero ha permitido exteriorizar mayor autonomía y reivindicación de sus derechos en una sociedad de orientación machista, generando como resultado mujeres más cuidadosas con las relaciones y con su fertilidad, por eso las familias han disminuido en número y aumentado su calidad de vida (Comisión Económica para América Latina y El Caribe [CEPAL-], 1994; Arriagada, 1997). Además, los distintos cambios familiares que individualizan la labor femenina han traído cambios dentro de las mismas porque de esta manera aumenta la oferta laboral donde las mujeres vienen ganando espacios (García y De Oliveira, 1994).

De acuerdo con Amarís (2011), las familias de doble ingreso procuran conciliar ambos mundos, sin tener la impresión de que haya un abandono por alguno de sus miembros, generando menores dificultades en la demanda tiempo, energía, cuidados, el trabajo y la alta productividad. Asimismo, se observa que cuando las mujeres llenan espacios laborales y poseen un adecuado reparto de los roles familiares y de pareja su labor del trabajo es de manera eficiente y se logra la salida de las tareas que históricamente le habían sido asignadas dentro del hogar, además, porque la actividad femenina extradoméstica no solo supone una doble jornada laboral, sino también una sobrecarga emocional y física (Googins y Burden, 1987; Caamaño, 2004).

Finalmente, se puede esbozar que la articulación entre vida familiar y laboral de las mujeres trae consigo el "incumplimiento" de la obligación prioritaria de cuidar los hijos y cubrir las necesidades de atención integral de la familia, considerándose una responsabilidad exclusivamente femenina (Googins y Burden, 1987). A propósito de las resistencias y los movimientos laborales y familiares logrados por las mujeres, estos han sido fundamentados en el hecho de que aún se puedan considerar roles asignados y segregados por sexos, ocasionando que los hombres no se sientan cómodos en "roles femeninos" porque desean conservar la tradición y se considere que los cambios en las familias son producto de la salida de la mujer al ámbito laboral, cuando realmente la transformación familiar atañe a lógicas sociales y no necesariamente al hecho de que los hombres aumenten su participación familiar (Pleck, 2014).

\section{Surgimiento de nuevas paternidades en el hogar}

Dentro de esta categoría se aborda el surgimiento de nuevas paternidades en el hogar, haciendo referencia a la participación masculina y resaltando a los hombres jóvenes como el principal modelo involucrado no solo en el cuidado de los hijos sino también en las actividades domésticas, sin dejar a un lado que este ha sido un "compromiso" generado para la mujer. 
Las nuevas paternidades en el hogar, de acuerdo con Macías et al. (2000), son aquellos cambios significativos en los entornos familiares que involucran la participación masculina y, aunque su papel continúa siendo secundario con un carácter de ayuda, el hombre ha empezado a asumir un rol cuidador y educativo, involucrándose en tareas de bienestar de los hijos.

Asimismo,Amarís (2011) y GómezyJiménez(2015)observan transformaciones en el modelo tradicional de relaciones entre hombres y mujeres con respecto al cuidado familiar, siendo los hombres jóvenes los principales interesados en apoyar en algunas tareas de atención y cuidado infantil, sin embargo, las tareas domésticas siguen siendo consideradas como propias de la mujer, de tal manera que es importante la existencia de cambios en los roles familiares, donde el hombre empieza a tener un mayor grado de participación en las actividades de cuidado. Sin embargo se aprecia, en relación a las tareas domésticas, que las mujeres continúan manteniendo su rol tradicional de amas de casa, así estén empleadas extralaboralmente.

A su vez, Gómez y Jiménez (2015) también plantean que cada vez más mujeres se vinculan a la esfera extradoméstica, y con ello se ha dado apertura a la construcción de roles familiares más democráticos, aceptando la participación de los hombres en la esfera familiar, sin embargo, aún se observa la influencia de creencias culturales sobre la responsabilidad del trabajo doméstico.

De otro lado, Bianchi (2000) afirma que, con la incorporación laboral femenina, el hombre se ha visto atraído a aumentar el tiempo destinado a la crianza de los hijos, situación que cobra especial valor si se tiene en cuenta que son las madres quienes ocupan grandes cantidades de tiempo a esa labor, incluso renunciando a otras actividades para criar a sus hijos. En comparación con otros tiempos, hoy los niños reciben tanto el tiempo del padre como de la madre, pero también se ha evidenciado que, de salir la mujer de su ámbito laboral remunerado, hay mayor involucramiento en lo familiar de lo masculino. Adicionalmente, tomando como referente la modernidad, en las generaciones de hombres más jóvenes se exterioriza más compromiso con aspectos de la paternidad como la crianza y el cuidado de los hijos (García y De Oliveira, 2001).

Siguiendo con las ideas de García y De Oliveira (2001), estos señalan que a nivel familiar se vienen generando un ejercicio de paternidad-maternidad compartida, aunque no es lo mismo en lo que se refiere a las actividades domésticas. Esta situación se encuentra caracterizada por conflictos debido a la escasa participación masculina en estas tareas.

En definitiva, no hay duda de que la incursión de las mujeres en el ámbito laboral ha venido incrementando los conocimientos y niveles educativos en el resto de la familia y ha permitido la redistribución de las actividades domésticas, que tradicional e históricamente han recaído sobre la mujer, promoviendo una implicación 
masculina en la resolución y/o redistribución de las tareas y actividades domésticas (Landwerlin, 1997).

Frente a estas nuevas paternidades, Torres (2014) plantea que los padres fueron modelados de una forma que no les permitió explorar diversas formas de paternidad, sin embargo, en las últimas décadas debido a los cuestionamientos de tipo social y cultural, estos padres se han visto abocados a una especie de paternidad democrática con nuevos valores y aprendizajes de cercanía con los hijos.

\section{Discusión}

Para llevar a cabo este estudio se tomaron como referencia los avances alcanzados en investigación sobre la creciente incorporación extralaboral de las mujeres y las transformaciones que esta ha propiciado en la vida familiar, permitiendo retomar elementos significativos acerca del papel que ha jugado la figura femenina en la familia durante las últimas décadas, y si bien es cierto que numerosos cambios sociales la han puesto en el ámbito extradoméstico, esto a su vez ha provocado transformaciones significativas en cómo se relacionan las familias.

Tomando como base los cambios de las últimas décadas, la temporalidad de 1980 a 2018 en este texto es muy relevante, debido a que coincide con la segunda ola del feminismo o feminismo de la liberación, donde las mujeres en Latinoamérica y por supuesto Colombia empezaron un proceso de autoconciencia hacia afuera, contribuyendo en la creación de diferentes propuestas y estrategias de divulgación a mujeres trabajadoras en lo rural y lo urbano, articulando fuerzas sociales y políticas orientadas hacía el cambio de prácticas con tintes excluyentes, discriminatorias y patriarcales (Lamus, 2010).

Frente a dichos cambios iniciados en esta segunda ola, la mayoría de los estudios coinciden en señalar las múltiples interacciones entre trabajo-mujerfamilia y resaltan la capacidad de cambio que proporciona la incorporación de la mujer al trabajo remunerado. Asimismo, ha recibido especial atención la discusión de los logros que permite o no impulsar el trabajo extradoméstico en términos de satisfacción por generar un ingreso económico adicional y contribuir en los gastos del hogar, sumado a realizar actividades fuera del ámbito doméstico que le generen independencia personal, económica y mayores espacios de negociación en el ámbito doméstico. Sin embargo, se identifican resistencias y eventuales tensiones familiares puesto que no hay una distribución en las actividades domésticas igualitarias, lo que puede generar mujeres en situaciones de agotamiento permanente, conllevando a dificultades en la convivencia, comunicación y relación de pareja a raíz de su trabajo interminable. Además, exigir condiciones más igualitarias, no solo con la presencia masculina en tareas de cuidado de los hijos sino también en los diferentes aspectos del ámbito doméstico. 
De igual manera, la investigación aportó elementos significativos para considerar. Por ejemplo, el trabajo extradoméstico como parte de un proyecto de desarrollo personal y profesional para las mujeres que genera autonomía y garantiza la participación en la toma de decisiones familiares, a su vez permitiéndole cuestionar patrones de autoridad e ir modificando el dominio masculino dentro del hogar (De Oliveira y Ariza, 1999; Abramo, 2004; García y De Oliveira, 2004).

A partir de estas consideraciones, vemos cómo la familia va camino a una transición del modelo tradicional de único proveedor masculino, quien asumía la máxima autoridad en las cuestiones importantes del hogar, para dar paso a otras formas de ordenamiento familiar, donde la mujer ha empezado a participar en decisiones reproductivas, económicas y de funcionamiento familiar, llevando a las familias a un liderazgo compartido que propicia un papel más activo de todos los integrantes de familia con la negociación de deberes.

En contraste, algunos autores constatan que más que el deseo por la superación personal, la necesidad e importancia del aporte económico de las mujeres al hogar proveniente del trabajo remunerado, siendo lo que las lleva a vincularse extralaboralmente, con el fin de contrarrestar dificultades económicas familiares o aumentar su poder adquisitivo para bienes y servicios que le generen a la familia mayor bienestar, calidad de vida o la disminución de la línea de pobreza (De Oliveira y Ariza, 1999; Guillén, 2007; Gómez y Jiménez, 2015), Además, que es justamente esta situación la que permite desarrollar ambientes más equitativos e igualitarios, puesto que en los hogares donde la contribución económica de la mujer es central o relevante en la manutención de la unidad doméstica, se garantiza su participación en la toma de decisiones y tiene mayor libertad de movimientos, mientras que las mujeres con menor contribución presentan frente a sus parejas menor autonomía y participación en las decisiones del hogar (Arriagada, 2004; García y De Oliveira, 2004).

En este mismo orden de discusión, las evidencias disponibles indican que, por lo general, las mujeres desarrollan múltiples estrategias para combinar las exigencias laborales y las responsabilidades del hogar, algunas de ellas buscan trabajos que sean compatibles, les permitan flexibilizar los horarios, tengan cercanía del hogar, modalidades de trabajo en casa, entre otros, con el fin de no descuidar sus funciones en el hogar y demostrar ser buenas madres, proveyendo protección permanente y segura para sus hijos (Rodríguez y Fernández, 2010). En el caso de las familias de doble ingreso, sigue siendo necesario conciliar ambos mundos, sin tener la impresión de que abandonan uno de ellos, resultando esta como una de las principales dificultades, dado que la familia demanda tiempo, energía, cuidados y el trabajo una alta atención y productividad.

Aun con todos estos cambios que ocurren con la participación de las mujeres en lo laboral, algunos autores han calificado como lento el modelo 
de repartos igualitarios que equilibre de forma consecuente los nuevos roles femeninos (Vega, 1994; White, 1999). En este sentido, hay que destacar que la participación de los esposos en las actividades domésticas es escasa cuando hay mujeres asalariadas y las transformaciones en la vida familiar no han alcanzado un nivel de igualdad, puesto que han estado acompañadas de una participación restringida de los hombres en este ámbito, conservando los roles tradicionales propios del género masculino, que les permite "ayudar" en lo doméstico, en lugar de compartir las responsabilidades, mientras que la mujer sigue haciéndose cargo de múltiples tareas del hogar y el hombre hace las reparaciones de la casa, o las tareas que les resultan más llevaderas y requieren menor grado de constancia, lo cual implica que las mujeres deban ejercer una doble carga laboral.

Por otra parte, varias de estas investigaciones han puesto de relieve el género como condicionante para el ingreso de la mujer al ámbito público, siendo evidente que para las mujeres el mundo laboral está más limitado que para los hombres; el hecho natural de convertirse en madres es uno de los principales determinantes para incorporarse a los espacios laborales. En el caso de los hijos en el hogar, es la razón fundamental por la que las mujeres llegan a considerar el reducir o eliminar el tiempo de trabajo productivo, en la búsqueda de conciliación de estos dos ámbitos, por lo cual se ha dado la conocida tendencia de aplazar o retrasar la decisión de formar hogar y de tener hijos, hasta no estar consolidadas profesional y laboralmente, para de esta manera garantizar que se concreten previamente aspectos del desarrollo personalprofesional. Dicha tendencia, trae consigo cambios a nivel familiar en lo que tiene que ver con su estructura, la diversificación de sus formas de unión y la disminución de fecundidad, que tienen una fuerte relación con la necesidad de las mujeres de conciliar su vida familiar y laboral (Amarís, 2011).

En cuanto a la categoría cambios tras resistencias, se encontró que autores como Vega (1994) y De Oliveira y Ariza (1999) se refieren a que la participación laboral para la mujer es de carácter opcional y cómo estos cambios han contribuido a provocar cambios en los papeles conyugales, llevando a la familia a replantearse las ideas que tienen sobre el modelo tradicional. En el caso de Sabater (2014), indica que esta transformación de los roles familiares y el aumento del empleo femenino obligan a buscar nuevas alternativas de organización. Este autor también señala que los cambios en los roles domésticos se producen después de un proceso de negociación dentro del hogar que se extiende a lo largo de diversos años e incluso de generaciones, así como que los hombres sufren un retraso adaptativo a esta nueva organización familiar y distribución de responsabilidades en el hogar. Adicionalmente, se encontró que uno de los cambios más representativos dentro de este tipo de dinámica familiar es que han disminuido las tasas de natalidad, y que esto se debe a que la mujer hoy por hoy retrasa su maternidad y antepone su vida laboral antes que una vida familiar, para así evitar los conflictos familiares que nacen del trabajo femenino remunerado. 
Se creería que no solo se trata de retrasar sino también de darse un lugar distinto a nivel personal y familiar, dado que esta nueva toma de decisiones sale de la esfera de lo tradicionalmente conocido para ellas.

También, se encontró que las mujeres aún guardan sentimientos de culpabilidad por no cumplir con las expectativas del modelo tradicional, esto es afirmado por White (1999), quien asegura que pueden continuar con sentimientos de culpabilidad por no responder a las expectativas tradicionales. Para disminuir esto, debe haber una división de roles y una reestructuración general familiar que permita la conciliación de estos dos ámbitos, esto teniendo en cuenta lo planteado por Fawaz y Soto (2012), quienes afirman que la progresiva participación femenina en el mercado laboral no va al mismo ritmo de la incursión de la mujer en el ámbito laboral.

En otro lugar, dentro de esta categoría de resistencia a cambios, se encontró que con el pasar de los años han surgido avances de igualdad y así nuevas distribuciones de las tareas en el hogar. Se refiere, específicamente, a la identificación de los factores que más contribuyen a la participación del hombre en la resolución de las tareas típicamente femeninas relacionando aspectos socioeconómicos como el hecho de que el grado de participación masculina varía también de forma apreciable según el nivel de ingresos de la mujer (Landwerlin, 1997). Respecto a esto, Amarís (2011) señala que una de las principales dificultades es que la familia demanda tiempo, energía, cuidados y el trabajo una alta atención y productividad.

También, se encontró que la actividad femenina extradoméstica no solo supone una doble jornada laboral, sino también una sobrecarga emocional y física, según Googins y Burden (1987), quienes agregan que estos cambios traen consigo el incumpliendo de la obligación prioritaria de cuidar los hijos y cubrir las necesidades de atención integral de la familia, considerado una responsabilidad exclusivamente femenina. En esa misma línea, Pleck (2014) afirma que estos cambios tienen su mayor incidencia en el rol de la esposa donde ella deba reducir su rol familiar que en el del hombre, dado que, por lo contrario, es poco creíble que aumente su rol en los aspectos familiares.

En cuanto a surgimiento de nuevas paternidades en el hogar, la revisión permitió encontrar que el rol del padre en familias con madres trabajadoras continúa siendo secundario con un carácter de ayuda. Autores como Gómez y Jiménez (2015) señalan que los hombres jóvenes son los principales interesados en apoyar en algunas tareas de atención y cuidado infantil, sin embargo, las tareas domésticas siguen siendo consideradas como propias de la mujer y, de igual manera, se ha visto que el rol de los hombres ha estado relacionado con el mantenimiento económico de la familia, enfatizando su rol de proveedor.

No obstante, en las nuevas sociedades se puede decir que el hombre ha aumentado el tiempo destinado a la crianza de los hijos, situación que cobra especial valor si tenemos en cuenta que son las madres quienes ocupan grandes cantidades de tiempo a esa labor. 
De acuerdo con Bianchi (2000) y García y De Oliveira (2001), las generaciones de hombres más jóvenes cada vez están más comprometidas con aspectos de la paternidad como la crianza y el cuidado de los hijos, además estos autores hacen énfasis en que estos cambios se vienen dando aparte de los conflictos relacionados con las actividades domésticas y la poca participación del hombre en estas. Esto coincide con Landwerlin (1997), quien manifiesta que no solo se observan conflictos en lo doméstico sino que también hay un aumento en el conocimiento y los niveles educativos en el resto de la familia que han contribuido en la redistribución de los trabajos en el hogar, que antes recaían sobre las mujeres, a esto se refiere un nuevo rol del hombre en el hogar.

\section{Conclusiones}

Los hallazgos permitieron visibilizar las distintas formas como las familias se transforman, reacomodan y sobreviven a los cambios generados en su exterior, denotando en su interior nuevas formas de organizarse, relacionarse y negociar, ante una de las variaciones más importantes que están atravesando las familias y que se relaciona con la creciente incorporación de las mujeres al empleo remunerado.

La participación de las mujeres en el mercado laboral contribuye a la construcción de relaciones familiares democráticas, en lo que tiene que ver con los procesos decisorios, de participación y consenso dentro de las familias y modificando ciertas prácticas relacionadas con modelos de tipo patriarcal. Este aspecto se relaciona con los planteamientos de Federici (2017), en tanto se expresa que las mujeres han promovido una reconstrucción de la realidad social a través de la economía feminista como nueva perspectiva que irrumpe en los paradigmas clásicos, económicos y dominantes criticando valores estructurales.

De igual forma, la mujer ahora participa en las decisiones familiares, tales como la disposición de ingresos propios y del hogar, consenso sobre la maternidad donde ambos cónyuges deciden sobre el número de hijos que desean, cuándo los desean y su crianza, así como cambios acerca de quién asume la máxima autoridad en cuestiones importantes, generando decisiones que favorecen la construcción de un balance en las relaciones conyugales y familiares.

La participación laboral de las mujeres genera aumento del bienestar económico familiar. Se observa que esta estrategia permite aliviar las condiciones de pobreza, contrarrestar dificultades económicas familiares o aumentar su poder adquisitivo para la consecución de bienes y servicios que le generen a la familia mayor bienestar y calidad de vida.

Sobre la distribución del trabajo doméstico no se observan cambios significativos en cuanto a la intervención de hombres, ya que estos solo "colaboran" con las tareas del hogar, pero la carga laboral de las mujeres se ha intensificado, puesto que además de trabajar extralaboralmente, continúan con toda la responsabilidad familiar. La 
doble jornada de trabajo constituye un aspecto común entre las mujeres asalariadas, puesto que se han convertido en proveedoras y continúan realizando las labores de cuidado y mantenimiento del hogar. En este lugar, es significativo rescatar el aporte de Torns (2005), dado que manifiesta que entre hombre y mujer hay desigualdades en la distribución del tiempo para ellas, proponiendo analizar el significado del tiempo que presentan las mujeres con relación a los hombres.

Sin embargo, es importante destacarse que si bien la participación masculina en las actividades domésticas es escasa en los hogares de mujeres asalariadas, encontramos un claro contraste en lo que tiene que ver con el cuidado de los hijos, dado que en los hogares en donde la madre trabaja extralaboralmente, la participación de los hombres es más frecuente; se ha producido un cambio de mentalidad sobre todo en los hombres más jóvenes que ahora consideran los deberes parentales y el cuidado de los hijos como una responsabilidad conjunta. Los padres han establecido mejores relaciones con sus hijos, hecho que contraste con la casi nula contribución en las labores domésticas. En palabras de Figueroa y Flores (2012), ha surgido un modelo alternativo de paternidad vinculado a los procesos de negociar y ejercer la autoridad, procurando un lugar de cuidado de los varones con sus hijos, que enseña y persuade a través del diálogo, dejando posturas de padre ausente o impositivo.

Todo esto permite concluir que una de las principales transformaciones que conlleva la incorporación de la mujer al ámbito laboral, permea elementos de socialización familiar, permitiendo visualizar los procesos de negociación y participación equitativa en laborales domésticas y extradomésticas, como contribución a la disminución de patrones de comportamiento patriarcales en las nuevas generaciones. Además, se proyecta una nueva forma de impulsar en los hijos un papel más activo dentro de los deberes de la familia sin distinción de género, que corrija desigualdades desde la niñez, introduciendo una imagen familiar y social positiva de la mujer que resignifica el trabajo doméstico y reconoce su participación en el ámbito público no solo como el intercambio de bienes y servicios necesarios para la sobrevivencia, sino también como contribución al proyecto de vida de la mujer y su familia.

\section{Referencias}

Abramo,L. (2004). ¿Inserción laboral de las mujeres en América Latina: una fuerza de trabajo secundaria? Revista Estudios Feministas, 12(2), 224-235. https://doi.org/10.1590/S0104-026X2004000200013

Alwin, D. F., Braun, M. y Scott, J. (1992). The separation of work and the family: Attitudes towards women's labour-force participation in Germany, Great Britain, and the United States. European Sociological Review, 8(1), 13-37.

Amarís, M. (2011). Roles parentales y el trabajo fuera del hogar. Psicología desde el Caribe, 15-28.

Arriagada, I. (1997). Realidades y mitos del trabajo femenino urbano en América Latina. 
Arriagada, I. (2004). Transformaciones sociales y demográficas de las familias latinoamericanas. Papeles de población, 10(40), 71-95.

Ayala, J. F., Cabezas, A. y Filippis, G. (2011). La integración de la mujer en el ámbito laboral. Poiésis, 11(22), 1-11.

Beal, X. V. (2011). ¿Cómo hacer investigación cualitativa? http://www.paginaspersonales.unam.mx/ files/981/94805617-Xavier-Vargas-B-COMO-HACER-INVESTIGA.pdf

Bianchi, S. M. (2000). Maternal employment and time with children: Dramatic change or surprising continuity? Demography (Pre-2011), 37(4), 401-14. https://search-proquestcom.ezproxy. uniminuto.edu/docview/222962399? accountid $=48797$

Bonaccorsi, N. (1999) El trabajo femenino en su doble dimensión: doméstico y asalariado. http://www. biblioteca.unlpam.edu.ar/pubpdf/aljaba/v04a06bonaccorsi.pdf

Caamaño, E. (2004). Conciliación de trabajo y vida familiar. Un análisis desde la perspectiva del derecho del trabajo chileno. Revista de derecho (Valdivia), 16, 59-82. https://dx.doi.org/10.4067/S071809502004000100003

Caballero, M. (2017). Entre el trabajo y la familia: Un estudio con dos parejas de doble ingreso. Criterio Libre Jurídico, 10(2) 25-34.

Cásares, E. (2008). La función de la mujer en la familia. Principales enfoques teóricos. Aposta: Revista de Ciencias Sociales, 36, 1-21.

CEPAL, (Comisión Económica para América Latina y el Caribe) (1994). La Cumbre Social: una visión desde América Latina y el Caribe. (LC/G.1802 (SES 25/5), Santiago de Chile.

Covarrubias, M. (2012). Maternidad, trabajo y familia: reflexiones de madres-padres de familias contemporáneas. La ventana, 4(35), 183-217.

De Oliveira, O. y Ariza, M. (1999). Trabajo, familia y condición femenina: una revisión de las principales perspectivas de análisis. Papeles de población, 5(20), 89-127.

Duarte, J. M. y García-Horta, J. B. (2016). Igualdad, Equidad de Género y Feminismo, una mirada histórica a la conquista de los derechos de las mujeres. Revista CS, 18, 107-158.

Engels, F. (1891). Origen de la familia, de la propiedad privada y del Estado. Editorial Roja.

Fawaz, J. y Soto, P. (2012). Mujer trabajo y familia: Tensiones, rupturas y continuidades en sectores rurales de Chile central. La ventana, 4(35), 218-254.

Federici, S. (2017). Economía feminista entre movimientos e instituciones: posibilidades, límites, contradicciones. En C. Carrasco y C. Díaz (Eds.). Economía feminista. Desafíos, propuestas, alianzas (pp. 21-28). Entrepueblos.

Figueroa, J. G. y Flores, N. (2012). Prácticas de cuidado y modelos emergentes en las relaciones de género. La experiencia de algunos varones mexicanos. La ventana, 35, 8-55.

García, B. y De Oliveira, O. (1994). Trabajo femenino, dinámica familiar y condición de la mujer: antecedentes y organización del estudio. En Trabajo femenino y vida familiar en México (pp. 19-38). El Colegio de México. https://www.jstor.org/stable/j.ctvhn0971.4? refreqid=excelsior\%3A7f663d2 ceb3bfce167fc1cc36ccce1db\&seq=4\#metadata_info_tab_contents

García, B. y De Oliveira, O. (2001). Cambios socioeconómicos y división del trabajo en las familias mexicanas. Investigación económica, 61(236), 137-162. 
García, B. y De Oliveira, O. (2004). Trabajo extradoméstico femenino y relaciones de género: una nueva mirada. Estudios Demográficos y Urbanos, 145-180.

García, B., Muñoz, H. y De Oliveira, O. (1982). Hogares y trabajadores en la Ciudad de México. El Colegio de México. doi: 10.2307/j.ctv26d9pv

Gómez, S. y Marti, C. (2004). La incorporación de la mujer al mercado laboral: Implicaciones personales, familiares y profesionales, y medidas estructurales de conciliación trabajo-familia (No. D/557). IESE Business School.

Gómez, V.y Jiménez, A. (2015). Corresponsabilidad familiar y el equilibrio trabajo-familia: medios para mejorar la equidad de género. Polis, Revista Latinoamericana, 14(40), 1-15.

González, A. (2008). La familia. Una mirada desde la Psicología. Medisur, 6(1), 4-13.

Googins, B. y Burden, D. (1987). Vulnerability of working parents: Balancing work and home roles. Social Work, 32(4), 295-300.

Guillén, N. (2007). Transformaciones en las familias: análisis conceptual y hechos de la realidad. Revista de Ciencias Sociales, (116), 35-56. https://revistas.ucr.ac.cr/index.php/sociales/article/ view/11192/10553

Haller, M. y Hoellinger, F. (1994). Female employment and the change of gender roles: The conflictual relationship between participation and attitudes in international comparison. International Sociology, 9(1), 87-112.

Labarge, M. W. (2003). La mujer en la Edad Media (Vol. 16). Editorial Nerea.

Lamus, D. (2010). De la subversión a la inclusión: movimientos de mujeres de la segunda ola en Colombia, 1975-2005. Instituto Colombiano de Antropología e Historia.

Landwerlin, G. (1997). La redefinición de la división del trabajo doméstico en la nueva familia urbana española. Reis, (80), 69-93. doi: 10.2307/40183917.

Macías, M. A., Camacho P. R. y Fernández, S. I. (2000). El rol del padre en las

familias que trabajan fuera del hogar. Psicología desde el caribe, (5), 157-175.

Mato, D. (1999). Cultura y transformaciones sociales en tiempos de globalización. Papel de Trabajo presentado en la 1 ra reunión del Grupo de Trabajo Cultura y transformaciones sociales en tiempos de globalización. Clacso.

Medina, J. A., Montero, A. C. y Sánchez, F. R. (2005). Las políticas de conciliación de la vida familiar y laboral en las Comunidades Autónomas. Cuadernos de relaciones laborales, 23(1), 73-93.

Navarro, A. (2010). ¿Mujeres proveedoras y jefas de familia? ...Nuevas realidades rurales en localidades de la región zamorana. La ventana, 4(31), 139-171. https://www.redalyc.org/articulo. oa? $\mathrm{id}=884 / 88415215007$

Pérez-Baleón, G. F. (2012). Desigualdades de género en el inicio de la vida laboral estable. Papeles de población, 18(72), 213-246.

Philip, T. (2002). Impact of women's employment on family and marriage: A survey of literature. Social Change, 32(1-2), 46-57.

Pleck, J. (2014). The Work-Family Role System. Social Problems, 24(4), 417-427. https://doi. org/10.2307/800135 
Ribeiro,M. (2004). Relaciones de género: equilibrio entre las responsabilidades familiares y profesionales. Papeles de población, 10(39), 219-237.

Rincón, A. (1995). Transformaciones en el papel social de las mujeres. Análisis cualitativo en Euskadi. Emakunde.

Rodríguez, M. del C. y Fernández, C. M. (2010). Empleo y maternidad: El discurso femenino sobre las dificultades para conciliar familia y trabajo. Cuadernos de Relaciones Laborales, 28(2), 257-275. https://search-proquestcom.ezproxy.uniminuto.edu/docview/853265156?accountid=48797

Romero, M. S. (2018). Arqueología feminista: desnaturalizando la subordinación. Entrevista con Margarita Sánchez Romero. ArkeoGazte, 8, 183-189.

Sabater, M. C. (2014). La interacción trabajo-familia. La mujer y la dificultad de la conciliación laboral. Lan harremanak: Revista de relaciones laborales, (30), 163-198.

Santelices, M. P., Besoain, C. y Escobar, M. J. (2015). Monoparentalidad, trabajo materno y desarrollo psicomotor infantil: Un estudio chileno en niños que asisten a salas cuna en contexto de pobreza. Universitas Psychologica, 14(2), 675-684.

Spitze, G. (1988). Women's employment and family relations: A review. Journal of Marriage and the Family, 50(3), 595-618.

Tirado, D. A., Muñoz, R. V. y Bravo, M. B. (2016). Conflicto familia-trabajo: dilemas y conflictos de mujeres trabajadoras en Las Comunas de Paillaco y Purranque, Sur de Chile. Cuaderno de trabajo social, (8), 9-25.

Tobio, M. C. (1998). Roles de género y la relación familia-empleo. Asparkia, IX, 22-44. Recuperado de https://e-archivo.uc3m.es/bitstream/handle/10016/20205/roles_TOBIO_AIF_1998.pdf?sequence=1

Torres L. (2004). La paternidad: una mirada retrospectiva. Revista de Ciencias Sociales, 3(105), 47-58.

Torns, T. (2005). El tiempo de trabajo y las relaciones de género: las dificultades de un cambio ineludible. Trabajo presentado en el Coloquio “Tiempos, actividades, sujetos”, en Madrid, 18 de febrero.

Urrutia, V., Faúndez, O. y Contreras, C. (2017). Negociaciones en familia: Género, trabajo y cuidado en Chile. Estudos Feministas, 25(2), 661-682.

Urrutia, V. G. y Figueroa, A. J. (2015). Corresponsabilidad familiar y el equilibrio trabajo-familia: medios para mejorar la equidad de género. Polis, Revista Latinoamericana, 14(40), 1-15.

Valdivia, S. C. (2008) La familia: concepto, cambios y nuevos modelos. La reevue du REDIF, (1), 15-22.

Vargas,X. (2011).¿Cómo hacer investigación cualitativo?. Recuperado de http://www.paginaspersonales. unam.mx/files/981/94805617-Xavier-Vargas-B-COMO-HACER-INVESTIGA.pdf

Vargas, G. (1988). Algunas características epistemológicas de la investigación documental. Revista de Ascolbi, 1(3), 26-33.

Vega, P. (1994). Trabajo productivo de la mujer y cambio en los roles familiares. El caso de Costa Rica. Anuario de estudios centroamericanos, 133-151.

Veneros, D. y Ortega, L. (2011). Trabajo femenino fabril en un contexto de modernización: Una visión de su evolución por provincias. Chile, 1910-1930. Universum (Talca), 26(1), 151-168.

White,J.(1999).Work-Family Stage and Satisfaction with Work-Family Balance. Journal of Comparative Family Studies, 30(2), 163-175. 